OPEN ACCESS

Edited by:

Fabyano Fonseca Silva, Universidade Federal de Viçosa,

Brazil

Reviewed by: Jude Bond,

New South Wales Department of

Primary Industries, Australia Marcio Duarte,

Universidade Federal de Viçosa,

Brazil

*Correspondence: Weimin Wang

wangwm@gsau.edu.cn

Specialty section:

This article was submitted to

Livestock Genomics,

a section of the journal

Frontiers in Genetics

Received: 21 June 2019 Accepted: 24 October 2019 Published: 19 November 2019

Citation:

Zhang D, Zhang X, Li F, Li C, La Y, Mo F, Li G, Zhang Y, Li X, Song $Q$, Zhao $Y$ and Wang $W$ (2019) Transcriptome Analysis Identifies Candidate Genes and Pathways Associated With Feed

Efficiency in Hu Sheep.

Front. Genet. 10:1183. doi: 10.3389/fgene.2019.01183

\section{Transcriptome Analysis Identifies Candidate Genes and Pathways Associated With Feed Efficiency in Hu Sheep}

\author{
Deyin Zhang ${ }^{1}$, Xiaoxue Zhang ${ }^{1,2}$, Fadi Li ${ }^{1,2,3}$, Chong $\mathrm{Li}^{1}$, Yongfu La', Futao Mo', Guoze Li', \\ Yukun Zhang ${ }^{1}$, Xiaolong Li ${ }^{1}$, Qizhi Song ${ }^{1}$, Yuan Zhao ${ }^{1}$ and Weimin Wang ${ }^{1 *}$ \\ ${ }^{1}$ College of Animal Science and Technology, Gansu Agricultural University, Lanzhou, China, ${ }^{2}$ Engineering Laboratory of \\ Sheep Breeding and Reproduction Biotechnology in Gansu Province, Minqin Zhongtian Sheep Industry Co. Ltd., Minqin, \\ China, ${ }^{3}$ The State Key Laboratory of Grassland Agro-ecosystems, College of Pastoral Agriculture Science and Technology, \\ Lanzhou University, Lanzhou, China
}

In the genetic improvement of livestock and poultry, residual feed intake (RFI) is an important economic trait. However, in sheep, the genetic regulatory mechanisms of RFI are unclear. In the present study, we measured the feed efficiency (FE)-related phenotypes of 137 male Hu lambs, and selected six lambs with very high $(n=3)$ and very low $(n=3) R F I$ values and analyzed their liver transcriptomes. A total of 101 differentially expressed genes were identified, of which 40 were upregulated and 61 were downregulated in the low-RFI group compared with that in the high-RFI group. The downregulated genes were mainly concentrated in immune function pathways, while the upregulated genes were mainly involved in energy metabolism pathways. Two differentially expressed genes, ADRA2A (encoding adrenoceptor alpha 2A) and RYR2 (ryanodine receptor 2), were selected as candidate genes for FE and subjected to single nucleotide polymorphism scanning and association analysis. Two synonymous mutations, ADRA2A g.1429 C > A and RYR2 g.1117 A > C, were detected, which were both significantly associated with the feed conversion rate. These findings provide a deeper understanding of the molecular mechanisms regulating FE, and reveal key genes and genetic variants that could be used to genetically improve FE in sheep.

Keywords: sheep, feed efficiency, residual feed intake, differentially expressed gene, single nucleotide polymorphism

\section{INTRODUCTION}

In indoor sheep production systems, feed represents $65-70 \%$ of the cost of production (Zhang et al., 2017). Residual feed intake (RFI) and feed conversion ratio (FCR) are two conventional indexes to measure feed efficiency. The FCR is defined as the ratio of weight gain to feed intake over a specific time, whereas RFI is the difference between the predicted and actual feed intake, adjusted for the body size and performance of each animal (Zhang et al., 2017). Low-RFI animals produce fewer pollutants and generate less waste (Zhang et al., 2017), but have no effect on the body size, production, and weight of the animals. Low-RFI animals not only protect the environment, but also reduce feed costs, making RFI an economically relevant trait. Understanding the molecular mechanism of RFI will help to breed sustainable and profitable animals in agriculture (De Oliveira et al., 2018). The liver is a central controller of metabolism and energy balances, and is a major driver 
of whole animal oxygen consumption in cattle, chickens, and pigs. The liver has potential roles in the control of feed efficiency variation (Alhusseini et al., 2016; Xu et al., 2016; Ramayo-Caldas et al., 2018).

Factors that affect RFI include the digestion and metabolism of nutrients, body composition, body activity, energy output, and body temperature regulation (Zhang et al., 2017). Among them, energy metabolism is an important factor. Genes controlling energy metabolism affect the FCR of animals by regulating the energy metabolism of the body (Mckenna et al., 2019). In recent years, studies on RFI candidate genes have focused on pigs, cattle, and poultry (Gondret et al., 2017; Mukiibi et al., 2018; Zeng et al., 2018). Some progress has been made in revealing the molecular mechanisms underlying the RFI phenotype. Pig skeletal muscle transcriptome analysis of messenger RNAs (mRNAs) and microRNAs demonstrated downregulation of genes involved in mitochondrial energy metabolism and upregulation of those involved in skeletal muscle differentiation and proliferation in low-RFI animals compared with those in high-RFI, animals (Jing et al., 2015). In brown-egg dwarf hens with extreme RFI phenotypes, the duodenal transcriptome architecture identified significantly differentially expressed genes (DEGs) that are involved in metabolism, digestibility, energy homeostasis, and biosynthesis (Yi et al., 2015). In pigs, genome-level analyses of candidate genes and genetic markers for RFI using genomewide associations and systematic genetic analyses revealed that single nucleotide polymorphisms (SNPs) in the genes encoding mitogen-activated protein kinase 5, peroxisomal biogenesis factor 7, and DS cell adhesion molecule could be markers associated with that for the RFI (Do et al., 2014). Gene functional annotation analysis suggested that the significant biological processes and pathways associated with RFI were gap junction formation, regulation of protein and lipid metabolism, the insulin signaling pathway, and inositol phosphate metabolism (Do et al., 2014). In Nellore cattle, a genome-wide association analysis of RFI and feed efficiency revealed markers located on chromosomes 4, 8, 14, and 21 in loci close to genes regulating ion transport and appetite (Santana et al., 2014). However, few studies have focused on the role of candidate genes in the process of RFI in sheep and as such, the genetic regulatory mechanisms of RFI are unclear.

In the present study, mRNA sequencing was used to determine the liver transcriptome and identify DEGs between sheep with extreme RFI values. We also explored the potential candidate genes that affect sheep RFI, which could lay the foundation for controlling in energy efficiency.

\section{MATERIALS AND METHODS}

\section{Ethics Statement}

The experiments performed in the present study were executed according to the approved guidelines from the Regulation of the Standing Committee of Gansu People's Congress. The Ethics Committee of Gansu Agriculture University approved all the experimental protocols and the collection of samples.

\section{Animals and Tissues}

In total, 598 male $\mathrm{Hu}$ lambs were purchased from Jinchang Zhongtian Sheep Industry Co. Ltd. (Jinchang, China) and transferred to Minqin Zhongtian Sheep Industry Co. Ltd. (Minqin, China). Healthy lambs at 56 days old, with good growth and available pedigrees, were selected randomly and immunized using a standardized program before weaning. The lambs were housed indoors in individual pens $(0.8 \times$ $1 \mathrm{~m}$ ) until they were 180 days old. Briefly, the lambs were exposed to an acclimatization period of 14 days, during which the proportion of pellet feed in the diet was gradually increased by $7.1 \%$ per day, while the forage proportion was concurrently reduced, until they were only fed the pellet feed. The pre-test period was 10 days and the experimental period was 100 days, during which all the sheep were fed pellet feed, and had free access to food and water. Lambs were weighed in the morning before feeding, and at 0 and 100 days of the experimental recording period using calibrated electronic scales. Throughout the experiment the housing and feeding conditions, and the environment, were standardized. At the end of the experimental period, under the supervision of qualified veterinarians, blood samples $(5 \mathrm{ml})$ were taken from the jugular vein of each sheep in the morning for subsequent DNA extraction. DNA was extracted using an EasyPure Blood Genomic DNA Kit (TransGen Biotech, Beijing, China). DNA was then dissolved in Elution Buffer (10 mM Tris hydrochloride, $1 \mathrm{mM}$ ethylenediaminetetraacetic acid; $\mathrm{pH}$ 8.0) and stored at $20^{\circ} \mathrm{C}$.

We collected the phenotype data of the experimental population at three different time, including 137 in the first batch, 207 in the second batch, and 254 in the third batch. We selected three high RFI sheep and three low RFI sheep from the first batch (137 male Hu lambs) to perform RNA sequencing. However, to expand the sample size of association analysis population, the phenotype of the other batches were measured after RNA sequencing. Within each RFI group, the sheep showed no difference in initial body weight (Table 2). A liver sample from each animal was obtained within $30 \mathrm{~min}$ after slaughter. The liver samples were frozen immediately using liquid nitrogen and then stored at $-80^{\circ} \mathrm{C}$ before RNA isolation. All three batches were used for association analysis between genotypes and feed efficiency traits.

\section{Phenotypes}

The phenotype of RFI was calculated using a linear regression model, using data on average daily gain (ADG), dry matter intake, and mid-test metabolic body weight (MBW) records for all the sheep.

The basic model (Zhang et al., 2017) used was:

$$
\begin{aligned}
& \mathrm{Y}_{\mathrm{j}}=\beta_{0}+\beta_{1} \mathrm{MBW}_{\mathrm{j}}+\beta_{2} \mathrm{ADG}_{\mathrm{j}}+\mathrm{e}_{\mathrm{j}} \\
& \mathrm{ADG}=(\mathrm{FBW}-\mathrm{IBW}) / \mathrm{N} \\
& \mathrm{MBW}=[1 / 2 \times(\mathrm{FBW}+\mathrm{IBW})]^{0.75}
\end{aligned}
$$


TABLE 1 | Descriptive statistics of all the experimental animals.

\begin{tabular}{|c|c|c|c|c|c|}
\hline Trait & $\mathbf{N}$ & Min & Max & Mean & SD \\
\hline $\mathrm{FCR}$ ( $\mathrm{kg}$ of $\mathrm{Fl} / \mathrm{kg}$ of BW gain) & 137 & 3.63 & 7.09 & 4.90 & 0.59 \\
\hline RFI (kg/day) & 137 & -0.31 & 0.22 & 0.00 & 0.09 \\
\hline $\mathrm{FI}$ (kg/day) & 137 & 0.72 & 1.72 & 1.22 & 0.18 \\
\hline ADG (kg/day) & 137 & 0.17 & 0.33 & 0.25 & 0.03 \\
\hline Initial BW (kg) & 125 & 1.42 & 18.99 & 2.75 & 1.55 \\
\hline Final BW (kg) & 137 & 15.15 & 36.70 & 24.72 & 3.95 \\
\hline Metabolic BW (kg0.75) & 137 & 9.82 & 16.89 & 13.11 & 1.33 \\
\hline BW before slaughter (kg) & 137 & 29.15 & 53.20 & 40.37 & 4.48 \\
\hline
\end{tabular}

FCR, feed conversion ratio; RFI, residual feed intake; Fl, average daily feed intake over the assessed feeding period; ADG, average daily gain over the assessed feeding period; BW, body weight.

TABLE 2 | Performance of the male Hu lambs used in RNA sequencing.

\begin{tabular}{|c|c|c|c|}
\hline & High-RFI & Low-RFI & p-value \\
\hline$n$ & 3 & 3 & \\
\hline $\begin{array}{l}\text { FCR ( } \mathrm{kg} \text { of } \mathrm{Fl} / \mathrm{kg} \text { of BW } \\
\text { gain) }\end{array}$ & $5.62 \pm 0.38$ & $3.92 \pm 0.25$ & 0.004 \\
\hline RFI (kg/day) & $0.20 \pm 0.02$ & $-0.25 \pm 0.05$ & 0.001 \\
\hline FI (kg/day) & $1.48 \pm 0.14$ & $1.05 \pm 0.13$ & 0.019 \\
\hline ADG (kg/day) & $0.26 \pm 0.02$ & $0.27 \pm 0.02$ & 0.827 \\
\hline Initial BW (kg) & $25.43 \pm 3.75$ & $25.76 \pm 2.09$ & 0.900 \\
\hline Final BW (kg) & $38.60 \pm 4.35$ & $39.10 \pm 2.69$ & 0.875 \\
\hline Metabolic BW (kg $\left.{ }^{0.75}\right)$ & $13.44 \pm 1.28$ & $13.59 \pm 0.74$ & 0.878 \\
\hline BW before slaughter (kg) & $41.18 \pm 4.06$ & $42.36 \pm 1.98$ & 0.678 \\
\hline
\end{tabular}

RFI, residual feed intake; FCR, feed conversion ratio; Fl, average daily feed intake over the assessed feeding period; $A D G$, average daily gain over the assessed feeding period; BW, body weight. P-value as calculated by a t-test.

where $Y_{j}$ is the dry matter intake of the $j^{\text {th }}$ animal, $\beta_{0}$ is the regression intercept, $\beta_{1}$ is the regression coefficient on $\mathrm{MBW}, \beta_{2}$ is the regression coefficient on $\mathrm{ADG}$, and $\mathrm{e}_{\mathrm{j}}$ is the uncontrolled error of the $j^{\text {th }}$ animal, FBW is final body weight, IBW is the initial body weight, and $\mathrm{N}$ is the test duration (days).

\section{RNA Preparation and Sequencing}

The Trizol reagent (Invitrogen, Waltham, MA, USA) was used to extract total RNA, according to the manufacturer's protocols. For each RNA-sequencing (RNA-seq) sample, a RNA sequencing library was constructed using a TruSeq ${ }^{\circledR}$ Stranded Total RNA Sample Preparation kit (Illumina ${ }^{\circledR}$, San Diego, CA, USA), performed according to the kit's manual. After quality control of the libraries, a HiSeq2500 instrument (Illumina) was used to sequence all the libraries.

\section{Analyses of RNA-Seq Data}

First, fqall2std.pl was used to transform the RNA-Seq data from the Illumina fastq format to the standard Sanger fastq format. Next, the Tophat-Cufflinks pipeline (Beijing Novogene Science and Technology Co., Ltd) was used to process the data. The reference genome of Ovis aries (Oarv4.0) and gene model annotation files were obtained from Ensembl. The reads were aligned to the genome using the build index with bowtie version 2.0.6. and TOPHAT (version 2.0.9), using the option: librarytype fr-firststrand. Transcriptome assembly was performed using Cufflinks (version 2.1.1), and gene expression analysis was executed using the Cuffdiff script (in Cufflinks) with the option: classic-fpkm. The fragments per kilobase of exon per million fragments mapped value was calculated to represent the expression level of each gene, based on the fragment length and read counts that mapped to a fragment. Finally, $\mathrm{q} \leq 0.05$ was set as the threshold for DEG selection. Quantitative real-time reverse transcription PCR (qRT-PCR) was used to validate the DEGs, we selected six sheep with the highest RFI (named the high-RFI group) and the six sheep with lowest RFI (low-RFI group) from the first batch (137 male Hu lambs) for extracting total RNA. Total complementary DNA (cDNA) was synthesized using a reverse transcriptase kit (Takara, Dalian, China). QPCR was performed using a SYBR green assay (Takara Biotechnology) on a Roche LightCycler 480 (Roche Applied Science, Mannheim, Germany). The specific quantitative primers for five transcripts are listed in Supplementary Material Table S1. Each 20- $\mu$ l reaction contained $6.4 \mu \mathrm{l}$ of $\mathrm{H}_{2} \mathrm{O}, 0.8 \mu \mathrm{l}$ of each primer, $2 \mu \mathrm{l}$ of cDNA, and $10 \mu \mathrm{l}$ of $2 \times$ SYBR Green PCR Master Mixture (Takara Biotechnology). The conditions were as follows: An initial single cycle $\left(95^{\circ} \mathrm{C}\right.$ for $\left.3 \mathrm{~min}\right)$, followed by 40 cycles $\left(95^{\circ} \mathrm{C}\right.$ for $15 \mathrm{~s}$; the optimized annealing temperature for $15 \mathrm{~s}$; and $72^{\circ} \mathrm{C}$ for $20 \mathrm{~s}$ ) and a final extension step at $72^{\circ} \mathrm{C}$ for $5 \mathrm{~min}$. All amplifications were followed by dissociation curve analysis of the amplified products. Gene expression levels were normalized to that of the ACTB gene (encoding beta actin) to determine the relative expression using the $2^{\Delta \Delta C t}$ value method. The average change in the cycle threshold $(\Delta \mathrm{Ct})$ value of the low-RFI group was used as the reference to calculate the $\Delta \Delta \mathrm{Ct}$ value for each gene (Livak and Schmittgen, 2001), and the expression difference between the two groups was calculated using Student's t-test.

\section{Kyoto Encyclopedia of Genes and Genomes Enrichment Analysis}

The Kyoto Encyclopedia of Genes and Genomes (KEGG) database contains resources that allow us understand gene functions in a biological system, for example at the cell, organism, and ecosystem levels. KEGG uses molecular level information, especially that generated by high-throughput experimental technologies, such as the large-scale molecular datasets generated by genome sequencing (http://www.genome. $\mathrm{jp} / \mathrm{kegg} /$ ). The statistical enrichment of DEGs in KEGG pathways was tested using the KOBAS software. 


\section{Single Nucleotide Polymorphism Identification and Genotyping}

We selected two genes (ADAR2A and RYR2) related to adrenaline pathway from 101 DEGs as candidate genes for SNP scanning. The SNPs in these two genes were identified by sequencing the PCR products that were amplified using mixed DNA samples from the Hu Sheep. The genomic DNA sequences of the candidate genes were used to design specific PCR primers (Supplementary Material Table S2). The PCR reaction for sequencing was performed in a volume of $25 \mu$, containing $10 \times$ PCR buffer, $0.35 \mu \mathrm{M}$ primers, $87.5 \mu \mathrm{M}$ dNTPs, $50 \mathrm{ng}$ of genomic DNA, and 1.25 of UTaq DNA Polymerase (TransGen Biotech) using the following thermocycling conditions: $5 \mathrm{~min}$ at $94^{\circ} \mathrm{C}$, followed by $30 \mathrm{~s}$ at $94^{\circ} \mathrm{C}, 30 \mathrm{~s}$ at $50-60^{\circ} \mathrm{C}$, and $30 \mathrm{~s}$ at $72^{\circ} \mathrm{C}(35$ cycles), with a final extension incubation for $5 \mathrm{~min}$ at $72^{\circ} \mathrm{C}$. Finally, competitive allele specific FRET-based PCR (KASPar) assays (LGC Genomics, Hoddesdon, UK) were used to genotype the SNPs identified within the candidate genes according to a previously published method (Smith and Maughan, 2015). The primers used by KASPar are shown in Supplementary Material Table S3. KASPar genotyping was developed using competitive allele-specific PCR and allows bi-allelic SNP scoring. To the DNA samples, the universal KASP Master Mix and SNP-specific KASP Assay mix (LGC genomics) were added. At the end of the thermal cycling, an end-point fluorescent reading was carried out. For allelic discrimination, competitive annealing of two allele-specific forward primers was performed. Each primer had a unique tail sequence that corresponded to a distinct labeled FRET cassette in the Master Mix. One was labeled with a HEX ${ }^{\mathrm{TM}}$ dye and the other with a FAM ${ }^{\mathrm{TM}}$ dye (Faucher et al., 2016).

\section{Statistical Analysis}

The general linear model program was used to perform the association analysis between genotypes and FCR. The specific model was defined as follows:

$$
\begin{aligned}
& \mathrm{Y}_{\mathrm{ij}}=\mu+\mathrm{G}_{\mathrm{i}}+\mathrm{B}_{\mathrm{j}}+\varepsilon_{\mathrm{ij}} \\
& \mathrm{FCR}=\text { average daily feed intake/ADG. }
\end{aligned}
$$

In this model, $\mathrm{Y}_{\mathrm{ij}}$ represents the phenotypic observation of FCR, $\mu$ is the mean, $G_{i}$ is the effect of the $i_{\text {th }}$ genotypes, $B_{j}$ is the batch effect and $\varepsilon_{\mathrm{ij}}$ is the residual corresponding to the trait's observed value, $P<0.05$ was considered to indicate statistical significance. The boxplots of FCR for different genotypes were computed using the ggplot packages in the R software.

\section{RESULTS}

\section{Animal Performance Traits}

Male Hu lambs $(n=137)$ were grown from 56 to 180 days old. The individual body weight (BW) and FI were measured and the ADG, FCR, and RFI were calculated (Table 1). From this data, sub-groups were selected from this data that had low or high RFI values i.e., the low-RFI and high-RFI groups. Notably, there was no significant difference in ADG between the two groups (Table 2). The high-RFI group had RFI and FCR values of $0.20 \pm 0.02$ $(\mathrm{kg} /$ day) and $5.62 \pm 0.38$, respectively, compared with $-0.25 \pm$ 0.05 (Kg/day) and $3.92 \pm 0.25$ in the more efficient low-RFI group $(P<0.05$ for both FCR and RFI; Table 2$)$. In the low-RFI group, a reduced FI resulted in a lower FCR $(P=0.019)$. We also noted there was no significant difference in average MBW between the two groups. Similar results were obtained in the other batches.

\section{RNA Sequencing Data Mapping and Annotation}

From the livers of the low-RFI and high-RFI groups, six cDNA libraries were sequenced and Pearson's correlation coefficient among the samples ranged from 0.92 to 0.96 , indicating high similarity of the expression patterns between the samples (Figure 1). After sequencing, a total of 370,689,678 raw reads were obtained from the high-RFI group and 334,871,310 from the low-RFI group. To test the quality of the RNA-seq data, we performed a series of quality control analyses. First, the Q30 i.e., the percentage of the total number of bases with a Phred score greater than 30, of the reads in all samples ranged from 91.5 to 93.22\%. The average GC content of the six libraries was $50.24 \%$. After filtering out the adaptor sequences, empty sequences, and low-quality sequences, 352,944,368 (high-RFI), and 328,070,686 (low-RFI) clean reads were obtained (Supplementary Material Table S4). The clean reads were then used to perform a biological information analysis. The mapping rate to the $O$. aries reference genome (Oarv4.0) of the clean data was between 81.07 and 83.03\% for all six samples (Supplementary Material Table S5).

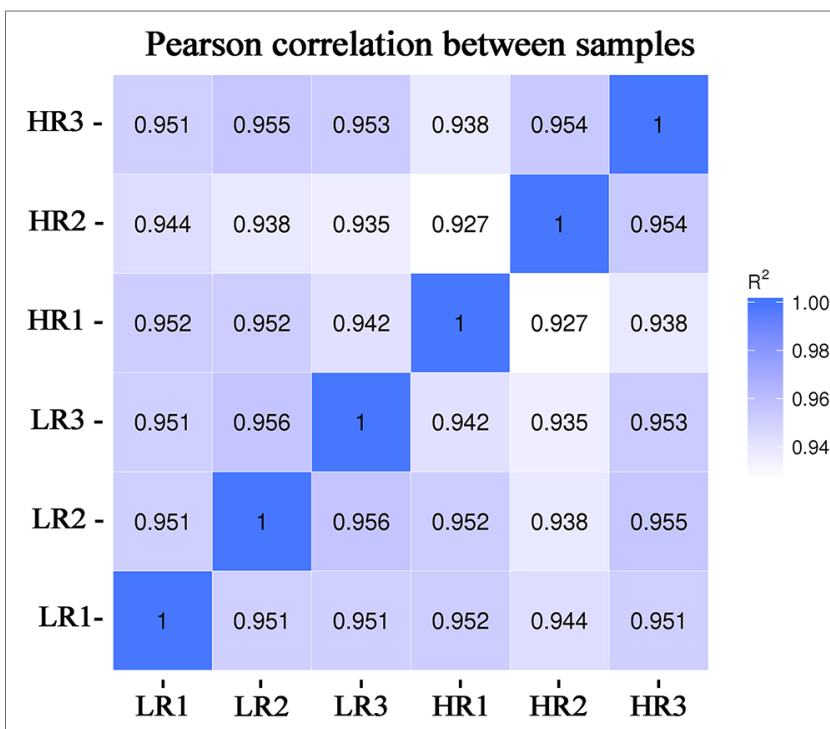

FIGURE 1 | Chart of the expression correlation between samples. Data for six samples, comprising three samples with very high-RFI values and three samples with very low-RFI values, are presented. The horizontal coordinate of the correlation coefficient between samples is $\log 10(F P K M+1)$ of sample 1 , the ordinate is $\log 10(F P K M+1)$ of sample 2 , and $R 2$ is the square of Pearson's correlation coefficient. FPKM-fragments per kilobase of exon per million fragments mapped. 


\section{Differentially Expressed Genes Between Low- and High-RFI Groups}

RNA-seq detected a total 22,801 genes in the livers of all six individuals, among which 744 genes were differentially expressed. DEGs were identified using the criteria of at least a 2-fold difference in expression and a p-value less than 0.05 $(|\log 2 \mathrm{FC}| \geq 1, P<0.05)$. Among the $744 \mathrm{DEGs}, 101$ had a $\mathrm{q}$-value $\leq 0.05$. Of the $101 \mathrm{DEGs}, 40$ were upregulated and 61 were downregulated in the low-RFI group (Supplementary Material Table S6). Table 3 shows the top 20 DEGs, representing the top 10 upregulated genes and the top 10 downregulated genes in the low-RFI group compared with that in the high-RFI group.

Five DEGs were selected for qRT-PCR analysis to validate their differential expression. The expression levels of $W W C 1$ (encoding WW and C2 domain containing 1), RFC3 (encoding replication factor $\mathrm{C}$ subunit 3 ), and $A D R A 2 A$ (encoding adrenoceptor alpha 2A) mRNA were lower in the low-RFI liver compared with that in the high-RFI liver, whereas the expression of SPP1 (encoding secreted phosphoprotein 1) and FCGBP (encoding Fc fragment of IgG binding protein) mRNA was higher in the low-RFI liver (Figure 2). The expression levels of five of the selected genes ( WWC1, RFC3, FCGBP, SPP1, and $A D R A 2 A)$ were significantly different between the high- and low-RFI groups, showing the qRT-PCR analyses confirmed the RNA-seq data.

\section{Pathway Analysis of Differentially Expressed Genes}

To further elucidate the functions of the DEGs, we performed KEGG pathway enrichment analyses. The KEGG pathway analysis of the DEGs between the low-RFI and high-RFI groups revealed that the downregulated genes were mainly involved in immune function pathways, including $D Q A$ (major histocompatibility complex, class II, DQ alpha 1), RFC3, RYR2 (ryanodine receptor 2), and PTGER3 (prostaglandin E receptor 3). The upregulated genes were mainly involved in metabolic pathways, including SPP1, FCGBP, PPARGC1A (PPARG coactivator 1 alpha), and $M E 1$ (malic enzyme 1) (Figure 3).

\section{Single Nucleotide Polymorphism Scanning of the ADRA2A and RYR2 Genes}

Two DEGs, ADRA2A and RYR2, were selected as candidate genes related to feed efficiency. Two synonymous mutations, g.1429 $\mathrm{C}>\mathrm{A}$ and g.1117 $\mathrm{A}>\mathrm{C}$ were discovered in ovine the ADRA2A and RYR2 genes, respectively, by sequencing the PCR products, which were amplified using mixed $\mathrm{Hu}$ Sheep DNA samples (Figure 4). The genomic DNA of these two candidate genes was used to design specific PCR primers (Supplementary Material Table S2). These two SNPs were genotyped using KASPar assays, which generated three genotypes for both genes: AA, CC, and AC (Figure 5).

\section{Association Analysis of ADRA2A and RYR2 Genes With the Feed Efficiency}

The association of SNPs in the ADRA2A and RYR2 genes with the FCR in the enlarged $(\mathrm{n}=561)$ experimental population was studied. The result of the association analysis showed that the novel polymorphisms ADRA2A g.1429 C > A and RYR2 g.1117 A $>$ C were both significantly associated with FCR $(P<0.05$ or $P<0.01)$. Lambs carrying the CC genotype of the ADRA2A g.1429 $\mathrm{C}>\mathrm{A}$ mutation had significantly lower FCR than those carrying the AA and CA genotypes $(0.51$ and 0.47 , respectively; $P<0.01)$

TABLE 3 | Top 20 liver DEGs between male Hu lambs with low- and high-RFI values.

\begin{tabular}{|c|c|c|c|c|c|}
\hline Gene & \multicolumn{2}{|c|}{ FPKM } & $\log _{2}(\mathrm{FC})$ & q-value & Full name \\
\hline SPP1 & 3.43 & 0.33 & 3.38 & 1.53E-02 & Secreted phosphoprotein 1 \\
\hline FCGBP & 1.19 & 0.25 & 2.22 & 8.28E-03 & Fc fragment of lgG binding protein \\
\hline ACTG2 & 3.39 & 0.89 & 1.92 & 8.28E-03 & Actin, gamma 2, smooth muscle \\
\hline SLITRK4 & 1.41 & 0.38 & 1.88 & 4.38E-02 & SLIT and NTRK-like family, member 4 \\
\hline SHISA3 & 0.87 & 0.25 & 1.81 & 2.67E-02 & Shisa family member 3 \\
\hline ME1 & 8.48 & 2.43 & 1.81 & 8.28E-03 & Malic enzyme 1 \\
\hline PPARGC1A & 20.99 & 6.41 & 1.71 & 8.28E-03 & PPARG coactivator 1 alpha \\
\hline ADRA2A & 0.65 & 5.21 & -2.99 & 8.28E-03 & Adrenoceptor alpha 2A \\
\hline NRCAM & 0.59 & 3.06 & -2.38 & 8.28E-03 & Neuronal cell adhesion molecule \\
\hline WWC1 & 11.02 & 57.49 & -2.38 & 8.28E-03 & WW and C2 domain containing 1 \\
\hline TCAF2 & 3.40 & 15.43 & -2.18 & 8.28E-03 & TRPM8 channel-associated factor 2 \\
\hline PTGER3 & 1.29 & 5.47 & -2.08 & 2.16E-02 & Prostaglandin E receptor 3 \\
\hline RIMS1 & 0.61 & 2.34 & -1.94 & 2.16E-02 & Regulating synaptic membrane exocytosis 1 \\
\hline
\end{tabular}

$D E G$, differentially expressed gene; RFI, residual feed intake; $\log _{2} F C$, $\log _{2}(l o w$-RFI/high-RFI); FPKM, fragments per kilobase of exon per million fragments mapped. 


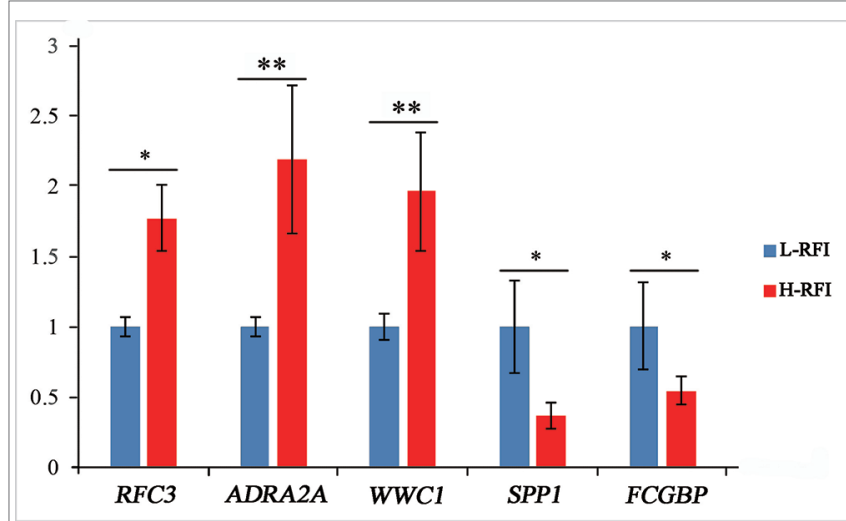

FIGURE 2 | Validation of differentially expressed genes (DEGs) using quantitative real-time reverse transcription PCR (qRT-PCR). The qRT-PCR measurements of the expression of RFC3, ADRA2A, WWC1, SPP1, and FCGBP mRNA transcripts were analyzed using the $\Delta \Delta$ Ct method; * significant difference between the high-RFI and low-RFI groups $(P<0.05)$, ${ }^{\star *}$ very significant difference between the high-RFI and low-RFI groups $(P<0.01)$.
(Table 4). Lambs carrying the AA genotype of the RYR2 g.1117 $\mathrm{A}>\mathrm{C}$ mutation had a significantly larger FCR than those carrying the $\mathrm{CC}$ and $\mathrm{CA}$ genotypes ( 0.33 and 0.38 , respectively; $P<0.05)$ (Table 4).

\section{DISCUSSION}

Two groups of male Hu lambs were phenotyped that had either high- or low-RFIs. The RFI, FI, and FCR values in the high-RFI lambs were significantly higher than those in the low-RFI lambs; however, there were no differences in the ADG, initial BW, final BW, MBW, and BW before slaughter, which was in agreement with the findings of Zhang et al. (2017). This result indicated that increased feed efficiency in sheep could result from the selection of RFIs by reducing feed consumption without affecting their growth performance. Therefore, RFI could be used as an ideal breeding index for feed efficiency traits in sheep.

The liver has a pivotal role in host physiology, carrying out most amino acid, carbohydrate, and lipid metabolism;
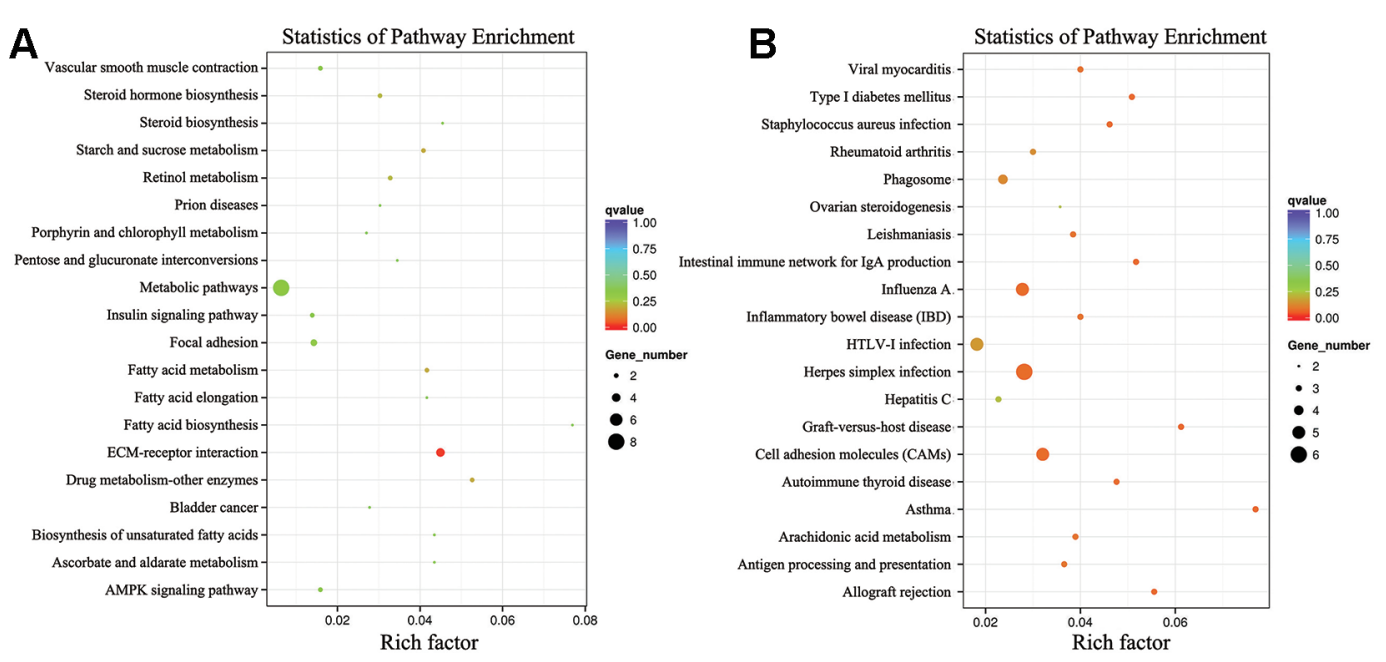

FIGURE 3 | Kyoto Encyclopedia of Genes and Genomes (KEGG) classification of differentially expressed genes (DEGs) between the high- and low-RFI groups. (A): Upregulated genes; (B): Downregulated genes.

\section{A ADRA2A g.1429 C $>$ A}

B $\quad R Y R 2$ g.1117 $\mathrm{A}>\mathrm{C}$
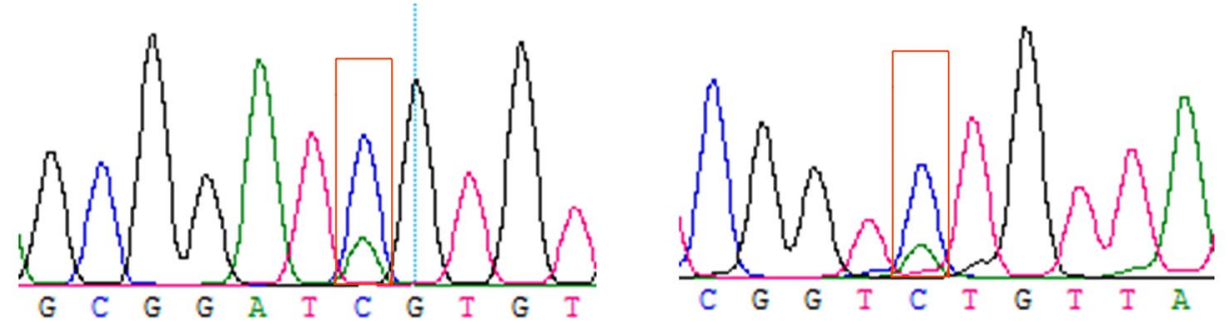

FIGURE 4 | Sequencing peak images for ovine ADRA2A (A) and RYR2 (B) genes. 
A

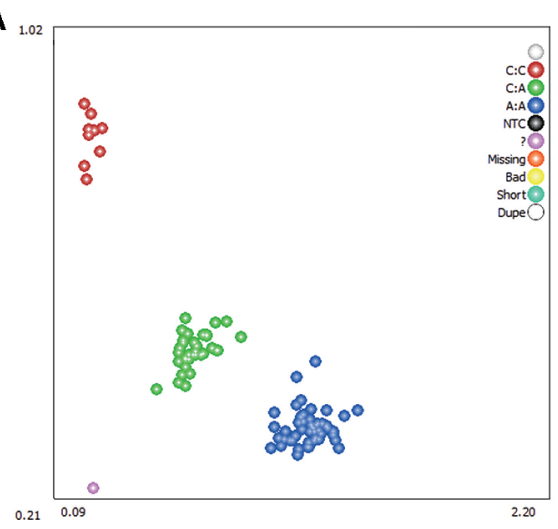

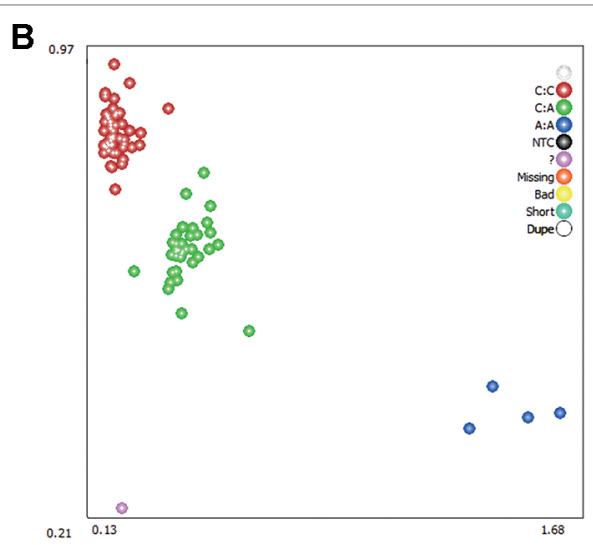

FIGURE 5 | Genotyping of ovine ADRA2A g.1429 C > A (A) and RYR2 g.1117 A > C (B) mutations using Kaspar technology. Note: The red, green, and blue dots represent the three genotypes, respectively; while the purple dots indicate genotyping failure.

TABLE 4 | Association analysis of ovine ADRA2A g.1429 C > A and RYR2 g.1117 A > C single nucleotide polymorphisms in the experimental population.

\begin{tabular}{lccc}
\hline Gene/loci & Genotype & No. & FCR \\
\hline ADRA2A g.1429 C > A & CC & 37 & $4.67 \pm 0.69 \mathrm{~B}$ \\
& CA & 215 & $5.18 \pm 0.92 \mathrm{~A}$ \\
RYR2 g.1117 A > C & AA & 309 & $5.14 \pm 0.93 \mathrm{~A}$ \\
& CC & 327 & $5.13 \pm 0.56 \mathrm{~b}$ \\
& CA & 207 & $5.08 \pm 0.65 \mathrm{~b}$ \\
& AA & 27 & $5.46 \pm 0.66 \mathrm{a}$ \\
\hline
\end{tabular}

Feed conversion rate (FCR) is indicated by the mean $+S D$. In the same column, there were significant differences between numerical data labeled with different lowercase letters $(P<$ 0.05), and between data labeled with different capital letters $(P<0.01)$.

detoxification; ketogenesis; urea synthesis; and albumin and glutathione synthesis; and is important in innate immunity (Rojas et al., 2015). In the present study, 101 DEGs were identified ( $\mathrm{q} \leq$ $0.05)$, including 61 downregulated genes between the low- and high-RFI animals, which are mainly concentrated in metabolic pathways. The results showed that metabolism-related genes were highly expressed in the liver tissues of the lambs with lowRFI, and the metabolic efficiency of the liver tissues of the lambs with low-RFI was improved, thereby improving feed efficiency.

The immune response process is another important factor affecting the efficiency of animal feed (Kyriazakis et al., 1998; Buyse and Decuypere, 2015). The immune system uses a lot of energy, for example, in mice that were immunized with a relatively benign antigen, metabolic heat production and oxygen consumption increased by $20-30 \%$ (Johnson et al., 2013). When an animal is diseased, the body will produce an immune response and the amount of food intake will be reduced. Immune defense and immune response are important mechanisms for animals to maintain their health. Energy metabolism is closely connected with productivity traits. The underlying mechanisms that control the immune defense or immune response may also affect the mechanisms that regulate energy metabolism (Verhagen, 1987). Liu et al. (2016) studied the whole blood expression profile of high and lowRFI pigs and identified genes involved in the immune system. In addition, Integrated Pathway Analysis Database-based disease-associated gene enrichment analysis suggested that genes involved in several diseases were overrepresented among the DEGs (Liu et al., 2016)(Liu et al., 2016). In the present study, 40 DEGs were upregulated between the low- and highRFI animals, which were mainly associated with immune pathways, including asthma, cell adhesion molecules, livestock influenza, the production of IgA intestinal immune network, type 1 diabetes, and inflammatory bowel disease, which showed that the high-RFI lambs had a heightened immune response. By stimulating the immune response, the body's energy consumption increases, thus lowering feed efficiency. Therefore, in the process of feeding, ensuring body health is an important basis to maximize the growth performance of animals.

Two DEGs, $A D R A 2 A$, and RYR2 participate in the adrenaline pathway and may regulate energy metabolism through the secretion of adrenaline, thus affecting feed efficiency. Therefore, the two genes were selected as candidate genes related to feed efficiency. Two synonymous mutations, g.1429 C > A and g.1117 A > C were discovered in the ovine $A D R A 2 A$ and $R Y R 2$ genes, respectively. Association analysis revealed that these two polymorphisms had a significant effect on feed efficiency. ADRA2A, a member of the $G$ protein-coupled receptor superfamily, regulates neurotransmitter release from adrenergic neurons and sympathetic nerves in the central nervous system (Manca et al., 1997; Fagerholm et al., 2004). ADRA2A is a regulator of catecholamines, which have been reported as associated with energy metabolism, and gene-regulated catecholamine release may play an important role in obesity (Lima et al., 2007). ADRA2A is also related to fat metabolism (Rosmond et al., 2010), and is implicated in several functions in the central nervous system, cardiovascular system, neurotransmitter release, platelet aggregation, blood pressure, insulin secretion, and lipolysis (Kaabi et al., 2016). Additionally, catecholamine-stimulated whole body lipolysis and lipolysis in subcutaneous adipocytes are blunted in obesity (Blaak et al., 1994; Large and Arner, 1998), thereby limiting lipid mobilization and favoring fat accumulation, which suggests that ADRA2A might be involved in fat and energy metabolism (Lima et al., 2007). Energy metabolism and fat metabolism are the key factors affecting the feed conversion rate of livestock and poultry (Kaewpila et al., 2018). However, the relationship between the 
$A D R A 2 A$ gene and feed efficiency in animals has not been reported previously. In our study, the $A D R A 2 A$ gene was downregulated in the low-RFI group. Moreover, polymorphism g.1429 C > A in this gene was significantly associated with FCR. Therefore, we speculated that $A D R A 2 A$ might affect FCR by changing energy metabolism and fat metabolism.

RYR2 is the main sarcoplasmic reticulum $\mathrm{Ca}^{2+}$ release channel in ventricular myocytes (Jiang et al., 2004). RYR2 regulation causes contractile dysfunction in a variety of cardiac pathologies (Bround et al., 2016). In particular, abnormal RYR2 activity contributes to sarcoplasmic reticulum $\mathrm{Ca}^{2+}$ mishandling, arrhythmias, and contractile dysfunction in failing hearts (Zima et al., 2014). In the present study, we found that $R Y R 2$ was downregulated in the lowRFI group, and that the gene has a $1117 \mathrm{~A}>\mathrm{C}$ mutation. Carrying the AA genotype of the RYR2 mutation resulted in a significantly larger FCR than that in those carrying the CC and CA genotypes. Therefore, RYR2, by regulating the excitement and contraction of skeletal muscle and cardiac muscle cells, affects the body's metabolic rate, which participates in the regulation of the sheep FCR; however, its regulation mechanism requires further study.

\section{CONCLUSIONS}

We identified 101 DEGs (40 upregulated and 61 downregulated) in liver of $\mathrm{Hu}$ lambs with different RFIs. Certain metabolism-related genes were upregulated between the high- and low-RFI groups, while downregulated genes were enriched in immune pathways. Importantly, two polymorphisms detected in DEGs ADAR2A and $R Y R 2$ were significantly associated with feed efficiency. These results provide new insights into the molecular mechanism of feed efficiency and identified valuable candidate genes for markerassisted selection to improve feed efficiency in sheep production.

\section{DATA AVAILABILITY STATEMENT}

The RNA-Seq Bioproject data are accessible at National Center for Biotechnology Information (NCBI) Bioproject under accession

\section{REFERENCES}

Alhusseini, W., Chen, Y., Gondro, C., Herd, R. M., Gibson, J. P., and Arthur, P. F. (2016). Characterization and profiling of liver microRNAs by RNA-sequencing in cattle divergently selected for residual feed intake. Asian-Australasian J. Anim. Sci. 29, 1371-1382. doi: 10.5713/ajas.15.0605

Blaak, E. E., Van Baak, M. A., Kemerink, G. J., Pakbiers, M. T., Heidendal, G. A., and Saris, W. H. (1994). Beta-adrenergic stimulation of energy expenditure and forearm skeletal muscle metabolism in lean and obese men. Am. J. Physiol. 267, E306. doi: 10.1152/ajpendo.1994.267.2.E306

Bround, M. J., Wambolt, R., Cen, H., Asghari, P., Albu, R. F., Han, J., et al. (2016). Cardiac Ryanodine Receptor (Ryr2)-mediated Calcium Signals Specifically Promote Glucose Oxidation via Pyruvate Dehydrogenase. J. Biol. Chem. 291, 23490-23505. doi: 10.1074/jbc.M116.756973

Buyse, J., and Decuypere, E. (2015). Chapter 19 - Adipose Tissue and Lipid Metabolism. Sturkies Avian Physiol. 443-453. doi: 10.1016/B978-0-12-407160-5.00019-1

De Oliveira, P. S. N., Coutinho, L. L., Tizioto, P. C., Cesar, A. S. M., De Oliveira, G. B., Diniz, W., et al. (2018). An integrative transcriptome analysis indicates number PRJNA547871. All RNA sequencing data have been submitted to the NCBI Sequence Read Archive under accession numbers SRR9291141, SRR9301148, SRR9301110, SRR9302163, SRR9302192, and SRR9302375.

\section{ETHICS STATEMENT}

The animal study was reviewed and approved by Standing Committee of Gansu People's Congress and The Ethics Committee of Gansu Agriculture University.

\section{AUTHOR CONTRIBUTIONS}

DZ and WW conceived the study. DZ, XZ, WW, FM, and YL contributed to growth performance and feed intake. DZ, XZ, FL, CL, GL, YKZ, XL, QS, YZ, and WW contributed to sample collection and prepared biological samples. DZ, XZ, and WW analyzed the data. DZ wrote the paper. DZ and WW revised the paper. All authors read and approved the final manuscript.

\section{FUNDING}

This work was supported by the National Natural Science Foundation of China (grant no. 31760651 and 31560625) and the Earmarked Fund for China Agriculture Research System (CARS-38).

\section{ACKNOWLEDGMENTS}

We would like to thank the native English-speaking scientists of Elixigen Company (Huntington Beach, California) for editing our manuscript.

\section{SUPPLEMENTARY MATERIAL}

The Supplementary Material for this article can be found online at: https://www.frontiersin.org/articles/10.3389/fgene.2019.01183/ full\#supplementary-material regulatory mRNA-miRNA networks for residual feed intake in Nelore cattle. Sci. Rep. 8, 1-12. doi: 10.1038/s41598-018-35315-5

Do, D. N., Strathe, A. B., Ostersen, T., Pant, S. D., and Kadarmideen, H. N. (2014). Genome-wide association and pathway analysis of feed efficiency in pigs reveal candidate genes and pathways for residual feed intake. Front. In Genet. 5, 307. doi: 10.3389/fgene.2014.00307

Fagerholm, V., Grönroos, T., Marjamäki, P., Viljanen, T., and Haaparanta, M. (2004). Altered glucose homeostasis in a 2A-adrenoceptor knockout mice. Eur. J. Pharmacol. 505, 243-252. doi: 10.1016/j.ejphar.2004.10.023

Faucher, L., Godé, C., and Arnaud, J.-F. O. (2016). Development of nuclear microsatellite loci and mitochondrial single nucleotide polymorphisms for the natterjack toad, Bufo (Epidalea) calamita (Bufonidae), using next generation sequencing and competitive allele specific PCR (KASPar). J. Heredity 107, 660. doi: 10.1093/jhered/esw068

Gondret, F., Vincent, A., Houée-Bigot, M., Siegel, A., Lagarrigue, S., Causeur, D., et al. (2017). A transcriptome multi-tissue analysis identifies biological pathways and genes associated with variations in feed efficiency of growing pigs. BMC Genomics 18, 244. doi: 10.1186/s12864-017-3639-0 
Jiang, D., Xiao, B., Yang, D., Wang, R., Choi, P., Zhang, L., et al. (2004). RyR2 mutations linked to ventricular tachycardia and sudden death reduce the threshold for store-overload-induced Ca2+ release (SOICR). Proc. Natl. Acad. Sci. 101, 13062-13067. doi: 10.1073/pnas.0402388101

Jing, L., Hou, Y., Wu, H., Miao, Y., Li, X., Cao, J., et al. (2015). Transcriptome analysis of mRNA and miRNA in skeletal muscle indicates an important network for differential Residual Feed Intake in pigs. Sci. Rep. 5, 11953. doi: 10.1038/srep11953

Johnson, K. A., Michal, J. J., Carstens, G. E., Hafla, A. N., and Forbes, T. D. A. (2013). Differential expression of innate immune system genes in liver of beef cattle with divergent phenotypes for RFI. Energy and protein metabolism and nutrition in sustainable animal production. (Wageningen Academic Publishers). doi: 10.3920/978-90-8686-781-3_130

Kaabi, B., Belaaloui, G., Benbrahim, W., Hamizi, K., Sadelaoud, M., Toumi, W., et al. (2016). ADRA2A Germline Gene Polymorphism is Associated to the Severity, but not to the Risk, of Breast Cancer. Pathol. Oncol. Res. 22, 357-365. doi: 10.1007/s12253-015-0010-0

Kaewpila, C., Sommart, K., and Mitsumori, M. (2018). Dietary fat sources affect feed intake, digestibility, rumen microbial populations, energy partition and methane emissions in different beef cattle genotypes. Animal 12, 1. doi: 10.1017/S1751731118000587

Kyriazakis, I., Tolkamp, B. J., and Hutchings, M. R. (1998). Towards a functional explanation for the occurrence of anorexia during parasitic infections. Anim. Behav. 56, 265-274. doi: 10.1006/anbe.1998.0761

Large, V., and Arner, P. J. D. (1998). Regulation of lipolysis in humans. Pathophysiological modulation in obesity, diabetes, and hyperlipidaemia. Diabetes Metab. 24, 409-418. doi: 10.1177/014572179802400611

Lima, J. J., Feng, H., Duckworth, L., Wang, J., Sylvester, J. E., Kissoon, N., et al. (2007). Association analyses of adrenergic receptor polymorphisms with obesity and metabolic alterations. Metabolism 56, 757-765. doi: 10.1016/j. metabol.2007.01.007

Liu, H., Nguyen, Y. T., Dan, N., Dekkers, J. C. M., and Tuggle, C. K. (2016). Postweaning blood transcriptomic differences between Yorkshire pigs divergently selected for residual feed intake. BMC Genomics 17, 73. doi: 10.1186/ s12864-016-2395-x

Livak, K. J., and Schmittgen, T. D. (2001). Analysis of Relative Gene Expression Data Using Real-Time Quantitative PCR and the $2-\Delta \Delta$ C T Method. Methods 25, 402-408. doi: 10.1006/meth.2001.1262

Manca, A., Volpi, E. V., Laficara, F., Muresu, R., Gray, I. C., Spurr, N. K., et al. (1997). Detailed Physical Analysis of a 1.5-Megabase YAC Contig Containing the MXI1 and ADRA2A Genes. Genomics 45, 407. doi: 10.1006/geno.1997.4924

Mckenna, C., Porter, R. K., Keogh, K. A., Waters, S. M., Mcgee, M., and Kenny, D. A. (2019). Residual feed intake phenotype and gender affect the expression of key genes of the lipogenesis pathway in subcutaneous adipose tissue of beef cattle. J. Anim. Sci. Biotechnol. 10, 212-221. doi: 10.1186/ s40104-018-0300-y

Mukiibi, R., Vinsky, M., Keogh, K. A., Fitzsimmons, C., Stothard, P., Waters, S. M., et al. (2018). Transcriptome analyses reveal reduced hepatic lipid synthesis and accumulation in more feed efficient beef cattle. Sci. Rep. 8, 7303. doi: 10.1038/ s41598-018-25605-3
Ramayo-Caldas, Y., Ballester, M., Sanchez, J. P., Gonzalez-Rodriguez, O., Revilla, M., Reyer, H., et al. (2018). Integrative approach using liver and duodenum RNA-Seq data identifies candidate genes and pathways associated with feed efficiency in pigs. Sci. Rep. 8, 558. doi: 10.1038/s41598-017-19072-5

Rojas, C.a.A., Ansell, B., Hall, R. S., Gasser, R. B., and Young, N. D. (2015). Transcriptional analysis identifies key genes involved in metabolism, fibrosis/ tissue repair and the immune response against Fasciola hepatica in sheep liver. Particle Fibre Toxicol. 8, 1-14. doi: 10.1186/s13071-015-0715-7

Rosmond, R., Bouchard, C., and Björntorp, P. (2010). A C-1291G polymorphism in the a2A-adrenergic receptor gene (ADRA2A) promoter is associated with cortisol escape from dexamethasone and elevated glucose levels. J. Internal Med. 251, 252-257. doi: 10.1046/j.1365-2796.2002.00961.x

Santana, M. H., Utsunomiya, Y. T., Neves, H. H., Gomes, R. C., Garcia, J. F., Fukumasu, H., et al. (2014). Genome-wide association analysis of feed intake and residual feed intake in Nellore cattle. BMC Genet. 15, 21. doi: 10.1186/1471-2156-15-21

Smith, S. M., and Maughan, P. J. (2015). SNP Genotyping Using KASPar Assays. Methods In Mol. Biol. 1245, 243. doi: 10.1007/978-1-4939-1966-6_18

Verhagen, J. M. F.Verhagen, J. M. F. (1987). Energy Metabolism and Immune Function. Energy Metabolism in Farm Animals. (Springer Netherlands). doi: 10.1007/978-94-009-3363-7_16

Xu, Z., Ji, C., Yan, Z., Zhe, Z., and Zhang, X. (2016). Combination analysis of genome-wide association and transcriptome sequencing of residual feed intake in quality chickens. BMC Genomics 17, 594. doi: 10.1186/s12864-016-2861-5

Yi, G., Yuan, J., Bi, H., Yan, W., Yang, N., and Qu, L. (2015). In-Depth Duodenal Transcriptome Survey in Chickens with Divergent Feed Efficiency Using RNASeq. PloS One 10, 136765. doi: 10.1371/journal.pone.0136765

Zeng, T., Zhang, H., Liu, J., Chen, L., Tian, Y., Shen, J., et al. (2018). Genetic parameters of feed efficiency traits and their relationships with egg quality traits in laying period of ducks. Poultry Sci. 97, 758-763. doi: 10.3382/ps/pex337

Zhang, X., Wang, W., Mo, F., La, Y., Li, C., and Li, F. (2017). Association of residual feed intake with growth and slaughtering performance, blood metabolism, and body composition in growing lambs. Sci. Rep. 7, 12681. doi: 10.1038/ s41598-017-13042-7

Zima, A. V., Bovo, E., Mazurek, S. R., Rochira, J. A., Li, W., and Terentyev, D. (2014). Ca handling during excitation-contraction coupling in heart failure. Pflügers Archiv - Eur. J. Physiol. 466, 1129-1137. doi: 10.1007/s00424-014-1469-3

Conflict of Interest: Authors XZ and FL were employed by company Minqin Zhongtian Sheep Industry Co. Ltd., China. The remaining authors declare that the research was conducted in the absence of any commercial or financial relationships that could be construed as a potential conflict of interest.

Copyright (C) 2019 Zhang, Zhang, Li, Li, La, Mo, Li, Zhang, Li, Song, Zhao and Wang. This is an open-access article distributed under the terms of the Creative Commons Attribution License (CC BY). The use, distribution or reproduction in other forums is permitted, provided the original author(s) and the copyright owner(s) are credited and that the original publication in this journal is cited, in accordance with accepted academic practice. No use, distribution or reproduction is permitted which does not comply with these terms. 